\title{
New Solutions to the Hierarchy Problem
}

\author{
Gustavo Burdman \\ Instituto de Física, Universidade de São Paulo \\ Cidade Universitaria, São Paulo, SP, 05508-900, Brazil
}

Received on 17 July, 2006

\begin{abstract}
After summarizing the status of the Standard Model, we focus on the Hierarchy Problem and why we believe this strongly suggests the need for new physics at the $\mathrm{TeV}$ scale. We then concentrate on theories with extra dimensions and their possible manifestations at this scale.
\end{abstract}

Keywords: Beyond the standard model; Electroweak bymmetry breaking; Extra dimensions

\section{INTRODUCTION}

The Standard Model (SM) is a very successful description of particle physics up to the weak scale. It agrees with all the experimental data we have our disposal today. However, it has several shortcomings. We do not know the origin of the fermion masses, which in the SM arise from the Yukawa interactions with the Higgs doublet. These are extremely varied, with a Yukawa coupling for the top of order one, and for the electron about $10^{6}$ times smaller. This constitutes the fermion mass hierarchy problem. Its solution might lie at high energy scales so that experiments at the $\mathrm{TeV}$ scale might not necessarily explore it. Another question unanswered by the SM, refers to the so called gauge hierarchy problem: why is the weak scale so much smaller than the Planck scale. We believe that the answer to this problem is at the $\mathrm{TeV}$ scale and therefore accessible to the Large Hadron Collider (LHC). We will first briefly summarize the status of the SM in Section II. In Section III we will expose the gauge hierarchy problem in more detail. In Section IV we will presents a review of solutions to the gauge hierarchy problem involving compact extra spatial dimensions.

\section{THE STANDARD MODEL}

The SM gauge group, $S U(2)_{L} \times U(1)_{Y}$, must be spontaneously broken to electromagnetism. This is achieved through the Higgs mechanism. The spontaneous breaking results in a set of 3 Nambu-Goldstone Bosons (NGB), which are not physical states since in the unitary gauge they are gauged away and appear as the longitudinal components of the $W^{ \pm}$ and the $Z^{0}$. Within the SM, a complex scalar doublet, corresponding to 4 degrees of freedom, results in one of them being left out, while the other are the NGBs. But in general, we can think of this as analogous to superconductivity: the Higgs mechanism results in a superconducting phase, where the order parameter of the phase is a scalar field, which may or may not be an elementary particle. As a result, the weak interactions become short range, just as electromagnetism becomes short range inside a superconductor, by getting an effective photon mass.

The first question is: at what scale should this phase transition occur ? In order to answer this question within the SM, we can look -for instance- at the scattering of massive gauge bosons. These amplitudes (without including the Higgs) grow like

$$
\frac{s}{M_{W}^{2}}
$$

violate unitarity unless something happens before $1 \mathrm{TeV}$. In the context of the SM, this means that we need $m_{h}<O(1) \mathrm{TeV}$ to restore unitarity.

The SM introduces the scalar doublet which has a Lagrangian:

$$
\mathcal{L}=\left(D_{\mu} \Phi\right)^{\dagger}\left(D^{\mu} \Phi\right)-V(\Phi)
$$

with the covariant derivative of the scalar doublet is given by

$$
D_{\mu} \Phi=\left(\partial_{\mu}+i g t^{a} W_{\mu}^{a}+\frac{i g^{\prime}}{2} B_{\mu}\right) \Phi
$$

The vacuum expectation value (VEV) $\left\langle\Phi^{T}\right\rangle=\left(\begin{array}{ll}0 & v / \sqrt{2}\end{array}\right)$ breaks the SM gauge group down to $U(1)_{\mathrm{EM}}$, leaving the photon massless and giving the weak gauge bosons masses:

$$
M_{W}=\frac{g v}{2} ; \quad M_{Z}=\frac{\sqrt{g^{2}+g^{\prime 2}} v}{2}
$$

The minimization of the Higgs potential results in $m_{h}=$ $\sqrt{2 \lambda} v$, with $\lambda$ the Higgs boson self-coupling.
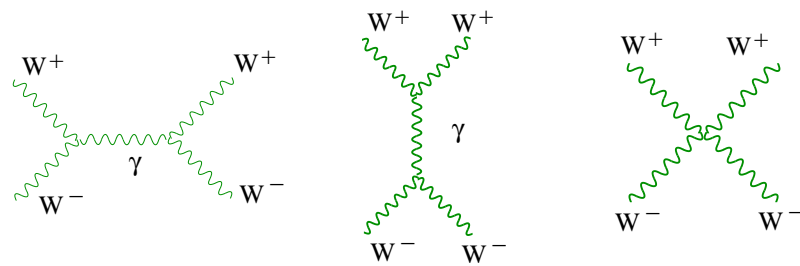

FIG. 1: Contributions to $W W$ scattering amplitude. It violates unitarity at about $1 \mathrm{TeV}$

The SM has been extremely successful when compared with experiment. The discovery of neutral currents in the 70's, and the discovery of the $W$ and $Z$ gauge bosons, where just the prelude to the spectacularly precise tests started in the 90's at LEP and SLAC. A large number of electroweak observables are predicted with the input of only three parameters. The fundamental parameters of the theory, $g, g^{\prime}$ and $v$, can be traded 


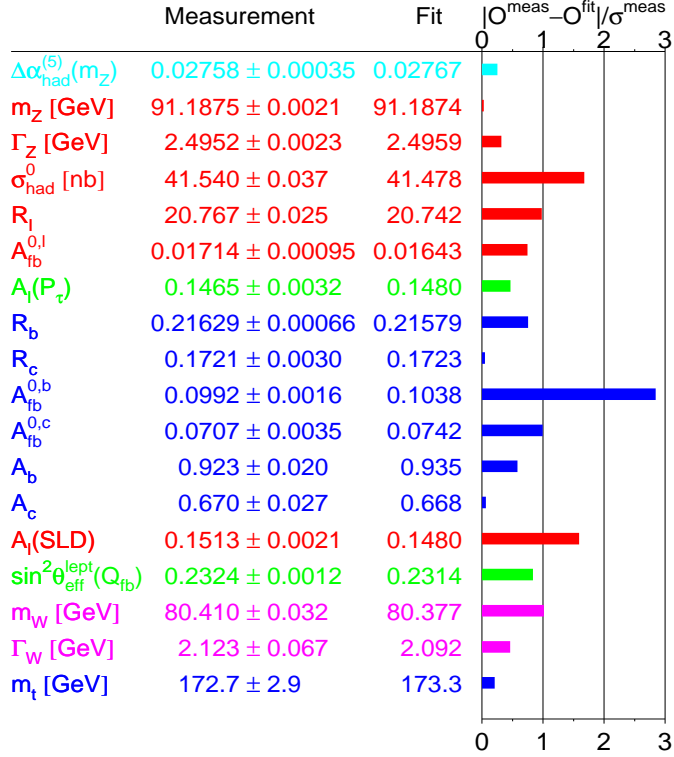

FIG. 2: Comparison of electroweak observables with the SM fit (Summer 2005)

by quantities that are more accurately known, typically $M_{Z}, \alpha$ and $G_{F}$. In Figure (2) we see a sample of the observables and the agreement with the SM fit is very good. The precision of the measurements has reach the sub-percent level, and in order to make predictions matching this one needs to go to one loop calculations. This means that the measurements are sensitive to heavy physics running around loops. The most important example is the Higgs itself. Since it has not been observed, been sensitive to its loop effects is very interesting. The sensitivity of the electroweak observables to the Higgs mass is only logarithmic. Yet, the precision is enough to turn this sensitivity into a bound on the Higgs mass. In Figure (3) we show the latest fit of the Higgs mass in the SM. The $\chi^{2}$ has a minimum at around $100 \mathrm{GeV}$, although the direct search bound is $m_{h}>114 \mathrm{GeV}$. The indirect limit implies that $m_{h} \leq 246 \mathrm{GeV}$ at $95 \%$ C.L. Thus, we conclude that the SM is in very good agreement with experiment and that the Higgs must be light.

Then why do we want to have physics beyond the SM ? The SM leaves many questions unanswered, and its answers to others are not very satisfactory. For instance, in the SM fermion masses arise as a consequence of Yukawa couplings to the Higgs. But since there is a huge hierarchy of masses (e.g. $m_{e} / m_{t} \simeq 10^{-6}$ ), then there should be a huge hierarchy of Yukawa couplings. This is the fermion mass hierarchy problem. The SM gauge group being a product group, plus the fact that the couplings get quite close to each other at high energies, suggest grand unification. What is the GUT group $G \subset S U(3)_{c} \times S U(2)_{L} \times U(1)_{\mathrm{Y}}$ ? What is the origin of the baryon asymmetry in the universe, or of dark matter and dark energy? Why is the cosmological constant so small ? So there is no shortage of shortcomings. However, we do not know at what energy scales these questions are answered. It could be

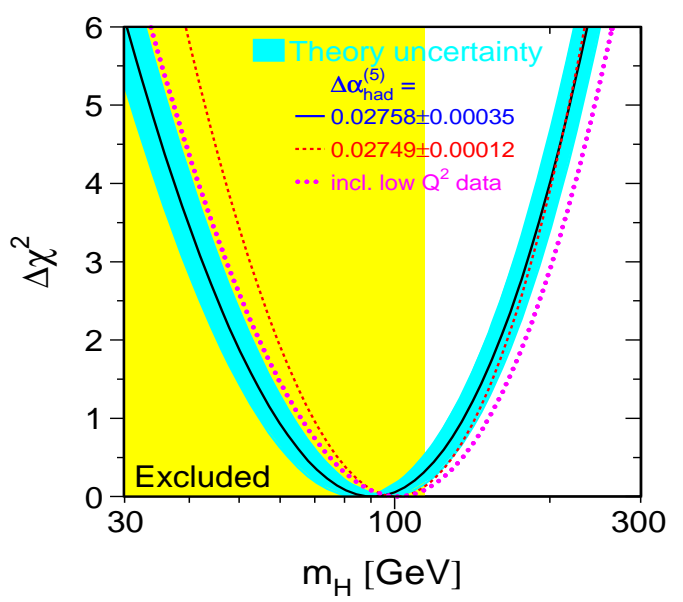

FIG. 3: The Higgs mass constraint in the SM.

that the physics associated with them lies at a scale unreachable experimentally, at least for the moment.

\section{THE HIERARCHY PROBLEM}

However, the question that requires physics beyond the $\mathrm{SM}$ at the $\mathrm{TeV}$, is the so called gauge hierarchy problem. One way to state it is simply by asking: why is the weak scale $(\simeq 100 \mathrm{GeV})$ so much smaller than the Planck scale $\left(\sim 10^{19} \mathrm{GeV}\right)$ ? The weak scale is given by the VEV of the Higgs, $v \simeq 246 \mathrm{GeV}$, the only dimensionfull parameter in the SM. However, it is not naturally stable under radiative corrections. If we consider the radiative corrections to the Higgs mass, coming from its couplings to gauge bosons, Yukawa couplings to fermions and its self-couplings, result in a quadratic sensitivity to the ultraviolet cutoff. Thus, if the SM were valid up to the Planck scale, then $m_{h}$ and therefore the minimum of the Higgs, potential, $v$, would be driven to the Planck scale by the radiative corrections. To avoid this, one has to adjust the Higgs bare mass in the SM Lagrangian to one part in $10^{17}$. This is quite unnatural, and is what we call the gauge hierarchy problem.

In order for physics beyond the SM to regulate the Higgs mass, and restore naturalness, its energy scale must be around the TeV. Several alternative theories are potential candidates to do this job. Most of them, imply that new physics will be discovered at the LHC. One of the most popular candidate theories is weak scale supersymmetry. The superpartners of the SM particles, having different statistics, contribute to the radiative corrections to the Higgs mass with the opposite sign. In the limit of exact supersymmetry, all corrections to $m_{h}$ cancel. However, since supersymmetry must be broken, there is a remnant logarithmic divergence dominated by the negative contribution of the top quark. Then, if the soft supersymmetry breaking scale is around $1 \mathrm{TeV}$, the Higgs becomes tachyonic and the electroweak symmetry is radiatively broken. This sce- 
nario, in its simplest incarnation, the minimal supersymmetric $\mathrm{SM}$, is considerably constrained to live in a fraction of its parameter space. The main constraint comes from the experimental lower bound on the Higgs mass. In the MSSM, $m_{h}$ is typically light, hardly above $120 \mathrm{GeV}$.

Another way to avoid the naturalness problem is not to have a Higgs at all, as in Technicolor (TC) theories. In these, a new strong interaction acting on techni-fermions, makes them condense breaking the electroweak symmetry. It runs into trouble when trying to generate fermion masses through extended TC interactions, also felt by the SM fermions. These interactions give rise to flavor changing neutral currents (FCNC). Raising the ETC energy scale may avoid the FCNC experimental bounds, but it makes very hard giving masses to heavier fermions, particularly the top quark. In order to address this, Topcolor interactions were introduced, giving rise to Topcolorassisted TC models. In addition to the fact that the picture, initially very appealing due to its simplicity, has gotten rather complicated, there are additional bounds on TC theories coming from electroweak precision constraints (EWPC) which tell us that QCD-like TC theories are practically ruled out.

If the Higgs boson is a composite state, and the compositeness scale is around the $\mathrm{TeV}$, then the corrections to its mass are cutoff at the TeV scale. Models of this kind present several problems, particularly with EWPC. However, if the Higgs is a pseudo-Nambu-Godstone boson, its mass is protected by some global symmetry. The gauge interactions explicitly break this symmetry giving rise to a Higgs mass. Little Higgs theories, proposed recently, are aimed at putting the cutoff of these strongly interacting theories, at a scale of tens of $\mathrm{TeV}$, whereas arranging for a collective symmetry mechanism that still protects $m_{h}$ from getting large. There are many choices of the global symmetry. In all cases, the gauge symmetry has to be extended beyond that of the SM, and new fermions must be introduced. Thus, these models have a very rich phenomenology at the LHC.

Finally, and also recently, the possibility of solving the hierarchy problem in theories with extra spatial dimensions has been considered. We will concentrate on these proposals in the rest of this presentation.

\section{EXTRA DIMENSIONS AND THE HIERARCHY PROBLEM}

Although theories with compact extra spatial dimensions have existed for quite some time, particularly in the context of string theory, it was only recently that extra dimensions have been invoked as a solution to the gauge hierarchy problem. Here we present three different scenarios where this is achieved.

\section{A. Large Extra Dimensions}

A new solution to the hierarchy problem involving extra spatial dimensions was proposed in Ref. [3]. In this setup,

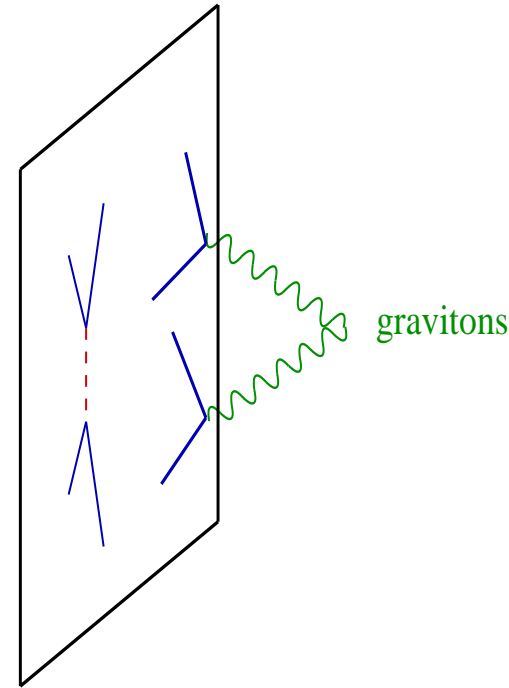

FIG. 4: Gravity propagating in the extra dimensional volume in the LED scenario. The SM matter and gauge fields are confined to a 4D slice, our brane.

there are $3+n$ spatial dimensions, with $n$ compact dimensions of a typical compactification radius $R$. All matter and gauge fields are confined to a four-dimensional slice, a brane. On the other hand, gravity can propagate in all $3+n$ dimensions, as shown in Figure (4). Therefore, gravity appears weak (i.e. $M_{P} \gg M_{W}$ ) because it propagates in the larger volume of compact extra dimensions, not available to the rest of the SM fields. The extra volume dilutes gravity's strength. The Planck scale is not a fundamental parameter in the extra-dimensional Einstein's action, but a scale derived from a volume suppression. The fundamental scale of gravity is $M_{*}$, and it satisfies

$$
M_{P}^{2}=M_{*}^{n+2} R^{n},
$$

where $n$ is the number of extra dimensions, and $R$ is the average compactification radius. Then, in principle, the fundamental scale of gravity could be much smaller than $M_{P}$ if the extra dimensions are big enough. For instance, if we have in mind solving the hierarchy problem, then we could choose $M_{*} \simeq 1 \mathrm{TeV}$. Then,

$$
R \sim 2 \cdot 10^{-17} 10^{\frac{32}{n}} \mathrm{~cm} .
$$

Thus, if we take $n=1$, we get that $R \sim 10^{8} \mathrm{Km}$, which is certainly excluded. Taking $n=2$, one has $R \sim 1 \mathrm{~mm}$, which is a distance scale already constrained by Cavendish-type experiments. For $n>2$, we need $R<10^{-6} \mathrm{~mm}$, which is not going to be reached by gravity experiments any time soon.

The fact that gravity propagates in compact extra dimensions leads to the existence of graviton excitations with a mass gap given by $\Delta m \sim 1 / R$. Then, in this scenario, there are new states, with spin 2 , and with rather small masses. For instance, for $n=2$ the Kaluza-Klein graviton mass starts at about $10^{-3} \mathrm{eV}$, and for $n=3$ at about $100 \mathrm{eV}$. Although the couplings of graviton excitations to matter are gravitationally suppressed, these states are so copiously produced at high energies $(E \gg 1 / R)$ that when we sum over all these final states, 
the inclusive cross sections are not suppressed by $M_{P}$, but by $M_{*}$ :

$$
\sigma \sim \frac{E^{n}}{M_{*}^{n+2}}
$$

On the other hand, since KK graviton lifetimes are still $M_{P}^{2}$ suppressed they would escape detection, leaving large missing energy signals as their mark. The processes that most uniquely would point to this physics at hadron colliders are of the mono-jet type, as depicted in Figure 5. Limits from
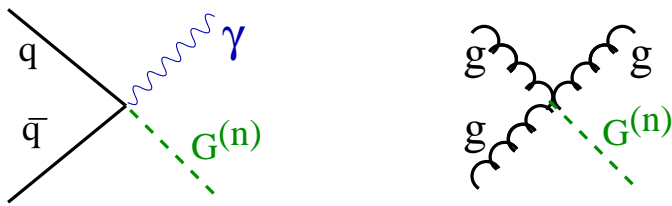

FIG. 5: Production of KK Gravitons.

the Tevatron already are available [6] and are already around $O(1) \mathrm{TeV}$, depending on the number of dimensions. Another signal of LED comes from the virtual exchange of KK gravitons. This induces dim- 6 operators of the form $\left(\bar{q} \gamma_{\mu} \gamma_{5} q\right)\left(\bar{f} \gamma_{\mu} \gamma_{5} f\right)$ entering, for example, in Drell-Yan production. Moreover, dim- 8 operators $\left(T_{\mu v} T^{\mu v}\right)$, result in $\bar{f} f \rightarrow$ $\gamma \gamma, Z Z, \cdots$. For $n=2$, the bounds on $M_{*}$ from the contributions of these operators are in the multi-TeV region already [6].

Astrophysical constraints have played an important role in the viability of the LED scenario. The most tight bound comes from Supernova cooling, where graviton KK emission could cool the supernova too fast. For instance, for $n=2$ this requires $M_{*}>(10-100) \mathrm{TeV}$.

Finally, the proposal that the fundamental scale of gravity, $M_{*}$, might not be far above the $\mathrm{TeV}$ scale, raises the possibility that strong gravity effects -such as black holes- might be visible at colliders experiments [7], or in ultra high energy cosmic rays [8].

\section{B. Universal Extra Dimensions}

If - unlike in the LED scenario - we allow fields other than the graviton to propagate in the extra dimensions, then constraints on $1 / R$ are much more severe. This is because the couplings of all other fields are not suppressed gravitationally, but at most by the weak scale. Naively, we would expect then that bounds on $1 / R$ climb rapidly to $O(1) \mathrm{TeV}$, and this is what happens [6] if some of the SM fields other than the graviton are in the bulk.

However, it was shown in Ref. [4] that if all fields propagate in the extra dimensional bulk (universal extra dimensions), then bounds on $1 / R$ drop to considerably lower values. The reason is that -upon compactification- momentum conservation leads to KK-number conservation. For instance, in 5D, $p_{5}$ the fifth component of the 5D momentum is quantified and given by

$$
p_{5}=n / R \text {, }
$$

with $n$ the KK number. The number $n$, (the fifth component of the momentum) must be conserved in interaction in the bulk. Thus, interactions involving two zero modes and one of the first excitations or $1 \mathrm{KK}$ mode, are forbidden. On the other hand, one zero mode can interact with two 1st excited states. This is illustrated in Figure (6). Among other things, this implies that in UED, KK modes cannot be singly produced, but they must be pair produced. This raises the bounds both from direct searches, as well as those from electroweak precision constraints [4]. Furthermore, compactification must be real-

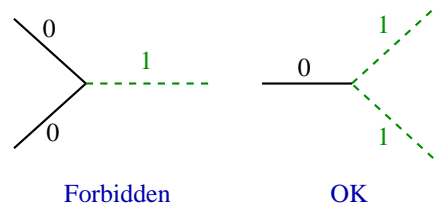

FIG. 6: Orbifold Compactification.

ized on an orbifold in order to allow for chiral fermion zeromodes, such as the ones we observe in the SM. In 5D, for example, this means an $S_{1} / Z_{2}$ compactification as illustrated in Figure 7. As a consequence of the orbifolding, KK-number
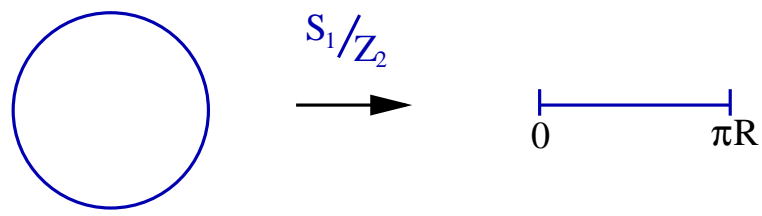

FIG. 7: Orbifold Compactification.

conservation is broken, but there is a remnant left: the parity of the KK modes must be conserved at the vertexes. This KKparity is very similar to R-parity in supersymmetric theories. It still means that zero-modes cannot be combined to produce a single KK excitation (for instance in an s-channel). In addition, KK-parity conservation means that the lightest state of the first KK level cannot decay into zero-modes, making it stable and a candidate for dark matter.

Direct constraints from the Tevatron in Run I as well as electroweak precision constraints on oblique parameters, give

$$
1 / R \geq\left\{\begin{array}{cc}
300 \mathrm{GeV} & \text { for } 5 \mathrm{D} \\
(400-600) \mathrm{GeV} & \text { for } 6 \mathrm{D}
\end{array}\right.
$$

These rather loose bounds imply that in principle even the Tevatron in Run II still has a chance of seeing UED. However, the signals are in general subtle. The reason is that -at leading order- all states in the same KK level are degenerate. Radiative corrections generate mass splittings [9], but these are still small enough for the energy release to be small in the production and subsequent decay of KK states. For instance, if a pair of level 1 quarks is produced, each of them might decay as $Q_{1 L} \rightarrow W_{1}^{ \pm} Q_{L}^{\prime}$, where the typical splitting between the $Q_{1 L}$ and the $W_{1}^{ \pm}$might be just a few tens of $\mathrm{GeV}$, depending on the number of extra dimensions (as well as the values of the brane kinetic terms). Thus, the quark jet tends to be soft, and this is 
repeated down the decay chain. In Ref. [10], the golden mode is identified as being $q \bar{q} \rightarrow Q_{1} Q_{1} \rightarrow Z_{1} Z_{1}+E_{T} \rightarrow 4 \ell+E_{T}$, where a large fraction of the missing energy is taken away by the lightest KK particle (LKP), in analogy with typical MSSM signals.

In fact, the similarity with the MSSM makes distinguishing it from UED a challenging proposition. The most distinct aspect of the UED scenario is the existence of KK levels, so the observation of the second KK level might be what is needed to tell it apart from other new physics [10]. In the $5 \mathrm{D}$ case, for instance, level $2 \mathrm{KK}$ states can decay via KKnumber conserving interactions to either another lighter level 2 state plus a zero-mode, or to two level 1 states. In both cases, the energy release is very small. On the other hand, localized kinetic terms induce not only additional mass splitting, but also KK-number violating (but KK-parity conserving) interactions. These are volume suppressed, and therefore there will only be able to compete so much with the phase-space suppressed terms mentioned above.

Six dimensional compactifications are also of interest. Unlike in the $5 \mathrm{D}$ case, in $6 \mathrm{D}$ the proton can be made naturally stable [11]. Also in 6D the number of fermion families must be three. In $6 \mathrm{D}$, however, there is more than one way of compactifying on an orbifold. We could have $T_{2} / Z_{2}$ or $T_{2} / Z_{4}$ compactifications. Recently, it was shown that an alternative way of folding the two compact extra dimensions, the chiral square, leads to the $T_{2} / Z_{4} \mathrm{KK}$ theory [12]. One can build gauge theories on the chiral square [13] and then derive in it the spectrum and couplings of a given theory [14], in order to study its phenomenology.

In the $6 \mathrm{D}$ case, the decay channel of the second $\mathrm{KK}$ level to two level 1 states is not present. This is because the generic mass of the second $\mathrm{KK}$ level is $M_{2}=\sqrt{2} / R$, which is smaller than $2 / R$, the sum of the masses of two level- 1 states. Thus, in the $6 \mathrm{D}$ scenario, level $2 \mathrm{KK}$ states can only decay through the localized kinetic terms and into two zero-modes. Furthermore, the signal also contains the contributions of the adjoint scalars, absent in 5D. These are the linear combinations of the 5th and 6th components of the gauge fields that are not eaten by the KK modes. They decay almost exclusively to top pairs [14]. These signals then may be used to distinguish the $5 \mathrm{D}$ and $6 \mathrm{D}$ cases. Then, the 6D scenario, well-motivated in its own merits (e.g. proton stability [11]) could be distinguished form the more typical MSSM-like 5D case.

\section{Warped Extra Dimensions}

A new solution to the hierarchy problem making use of one extra dimension was proposed in Ref. [5]. Unlike the two previous cases, the extra dimension does not have a flat metric. In the Randall-Sundrum (RS) setup, the metric is that of Antide Sitter in 5D, and is given by:

$$
d s^{2}=e^{-2 k|y|} \eta^{\mu v} d x_{\mu} d x_{v}+d y^{2},
$$

which is a solution of Einstein's equations in 5D, as long as the bulk cosmological constant is adjusted to cancel the cosmological constant on the fixed points. Then, the branes have a flat metric, as desired. In eqn.(10) $k \lesssim M_{P}$ is the $A d S_{5}$ curvature and $y$ is the coordinate of the fifth dimension. The only scale in the 5D Einstein-Hilbert action is $M_{P}$. However, when at a distance $y$ from the origin of the extra dimension, all energies are exponentially suppressed by a factor of $\exp (-k y)$. Then, if all SM fields, except gravity, were confined at a distance $L$ from the origin, the local cutoff would not be $M_{P}$ but

$$
\Lambda_{L}=M_{P} e^{-k L}
$$

This is depicted in Figure 8. The compactification is done in the $S_{1} / Z_{2}$ orbifold, with $L=\pi R$. If we want the local cutoff to

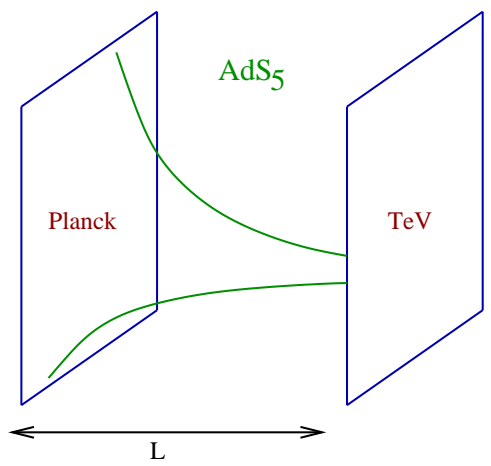

FIG. 8: Warped Extra Dimension. The local cutoff is exponentially smaller than $M_{P}$.

be the $\mathrm{TeV}$ scale, therefore explaining the hierarchy, we need to choose

$$
k R \simeq O(10)
$$

which does not constitute a very significant fine-tuning. Then, in the RS scenario, an exponential hierarchy is generated by the non-trivial geometry of the extra dimension. This scenario already has important experimental consequences. Since gravity propagates in the bulk, there is a tower of KK gravitons. The zero-mode graviton has a wave-function localized towards the Planck brane and couples to matter with its usual coupling, suppressed by $1 / M_{P}^{2}$. The KK gravitons, on the other hand, have masses starting at $\mathrm{O}(1) \mathrm{TeV}$, and couple to matter on the $\mathrm{TeV}$ brane as $1 /(\mathrm{TeV})^{2}$. Then, KK gravitons can be produced at accelerators with significant cross sections. For instance, the Drell-Yan process would receive a contribution from s-channel KK gravitons as in $q \bar{q} \rightarrow G^{(n)} \rightarrow e^{+} e^{-}$.

The RS proposal solves the hierarchy problem because the radiative corrections to $m_{h}$ are now cutoff at the TeV scale. The SM operates in our 4D slice. But the mechanism of EWSB is still that of the SM. Moreover, the origin of fermion masses (the other hierarchy) is completely unexplained, together with a number of other issues ranging from gauge coupling unification to dark matter. Allowing additional fields to propagate in the bulk opens up a great deal of model building opportunities. In general, unless supersymmetry is invoked, the Higgs must remain localized on or around the $\mathrm{TeV}$ brane, or it would receive large quadratically divergent corrections of order of $M_{P}$, just as in the SM. 
If gauge fields are allowed in the bulk then their KK expansion takes the form

$$
A_{\mu}(x, y)=\frac{1}{\sqrt{2 \pi R}} \sum_{n=0}^{\infty} A_{\mu}^{(n)}(x) \chi^{(n)}(y)
$$

where $\chi^{(n)}(y)$ is the wave-function in the extra dimension for the nth KK excitation of the gauge field. In the 4D effective theory, there is - in general - a zero-mode $A_{\mu}(x)^{(0)}$, and a KK tower of states with masses

$$
m_{n} \simeq(n-O(1)) \times \pi k e^{-k \pi R},
$$

starting at $O(1) \mathrm{TeV}$. Their wave-functions are localized towards the TeV brane. The gauge symmetry in the bulk must be enlarged with respect to the SM in order to contain an $S U(2)_{L} \times S U(2)_{R}$ symmetry. The extra $S U(2)_{R}$ restores a gauge version of custodial symmetry in the bulk, thus avoiding severe $T$ parameter constraints [16] (in the dual language of the CFT, there is a global symmetry associated with it, the custodial symmetry).

Just like the gauge fields, if fermions are allowed to propagate in the bulk they will have a similar KK decomposition, given by

$$
\Psi_{L, R}(x, y)=\frac{1}{\sqrt{2 \pi R}} \sum_{n=0} \psi_{n}^{L, R}(x) e^{2 k|y|} f_{n}^{L, R}(y),
$$

where $f_{n}^{L, R}(y)$ are the wave-functions of the KK fermions in the extra dimension, and the superscripts $L$ and $R$ indicate the chirality of the KK fermion. Since fermions are not chiral in $5 \mathrm{D}$, half of their components are projected out in the orbifold compactification. Unlike gauge fields in the bulk, fermions are allowed to have a mass term since there is no chiral symmetry protecting it. Then the typical, bulk fermion mass term looks like

$$
S_{f}=\int d^{4} x d y \sqrt{-g}\{\cdots-c k \bar{\Psi}(x, y) \Psi(x, y)\}
$$

where naturally $c \sim O(1)$, i.e. the bulk fermion mass is of the order of the AdS curvature scale $k$. The KK fermion wavefunctions in this case have the form

$$
f_{0}^{R, L}(y)=\sqrt{\frac{k \pi R(1 \pm 2 c)}{e^{k \pi R(1 \pm 2 c)}-1}} e^{ \pm c k y} .
$$

Then, the localization of the KK fermion wave function in the extra dimension is controlled by the $O(1)$ parameter $c$ with exponential sensitivity [17]. All that is needed to explain the wildly varying fermion spectrum is $O(1)$ flavor breaking in the bulk, which could be naturally originated at the cutoff. Fermions with wave-functions towards the $\mathrm{TeV}$ brane $(c<1 / 2)$ will have a larger overlap with the Higgs VEV, and therefore a larger mass, of $O(v)$. Light fermions, on the other hand, will have wave-functions localized towards the Planck brane $(c>1 / 2)$. For $c=1 / 2$ the fermion wave-function is flat. This is shown in Figure 9. The need to generate a large enough value for $m_{t}$, forces us to localize the top quark not

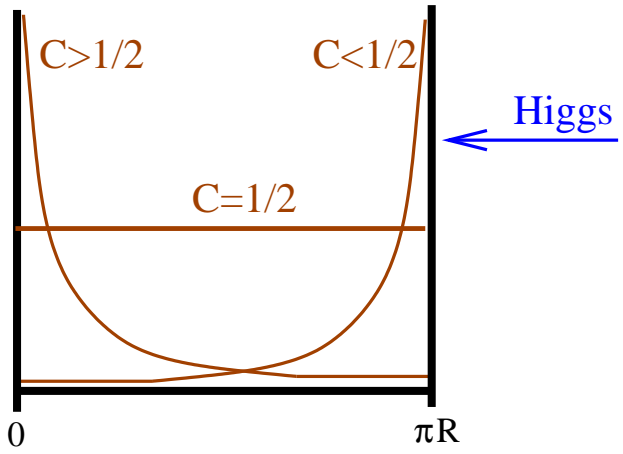

FIG. 9: Fermions are localized according to the choice of the $O(1)$ parameter $c$, the bulk fermion mass in units of $k$, the AdS curvature.

too far from the TeV brane. Even if we localize $t_{R}$ to this IR fixed point, the localization of $t_{L}$-and consequently $b_{L^{-}}$cannot be chosen to be at the Planck brane. If $b_{L}$ is forced to have a significant overlap with the $\mathrm{TeV}$ brane, there will be non-universal couplings of both the KK gauge bosons of all gauge fields, and the zero-mode weak gauge bosons $\left(W^{ \pm}, Z\right)$. The latter, result from the deformation of their (otherwise flat) wave-function due to the Higgs VEV on the TeV brane. Fermions with profiles close to the TeV brane can feel this effect and couplings like $Z \rightarrow b_{L} \bar{b}_{L}$ would be affected [16]. It would also lead to FCNCs at tree level mediated by the $Z$, and that could be observed in $B$ decays such as $b \rightarrow \ell^{+} \ell^{-}$[18]. On the other hand, the KK excitations of all gauge bosons would have non-universal couplings to all fermions, and particularly to the top and to $b_{L}$. This could lead to interesting effects in hadronic $B$ decays and $C P$ violation, especially when considering the interactions with KK gluons [19].

Finally, we could ask the question: could we get rid of the Higgs boson altogether ? After all, it looks a bit ad hoc, localized in the $\mathrm{TeV}$ brane. We know that boundary conditions (BC) can be used to break gauge symmetries in extra dimensional theories. It was proposed in Ref. [20] that the electroweak symmetry could be "broken" by BC in a 5D theory as a way to replace the Higgs field. The first question would be: what about the unitarity of electroweak amplitudes such as $W^{+} W^{-}$scattering ? If the Higgs boson is not present how are these amplitudes unitarized? The answer is that the KK excitations of the gauge bosons do the job [21]. The actual models that (nearly) work are similar to the one we had before on $A d S_{5}$, but without a Higgs boson on the TeV brane [22]. The $\mathrm{BC}$ can be thought of as obtained by the presence of branelocalized scalar fields that get VEVs. In the limit of these VEVs $\rightarrow \infty$, one recovers the BC. Thus although the origin of the $\mathrm{BC}$ might be a set of scalar fields getting VEVs, these need not be at low energies. It is in this sense that the theory is Higgsless.

The other question is how do fermions get their masses ? With the appropriate choice of BC the bulk gauge symmetry breaks as $S U(2)_{L} \times S U(2)_{R} \times U(1)_{X} \rightarrow U(1)_{\mathrm{EM}}$. But the $\mathrm{BC}$ restrict the gauge symmetry differently at different fixed points. This can be seen in Figure 10. The BC restrict the symmetry at the TeV brane to be $S U(2)_{L+R}=S U(2)_{V}$, which 


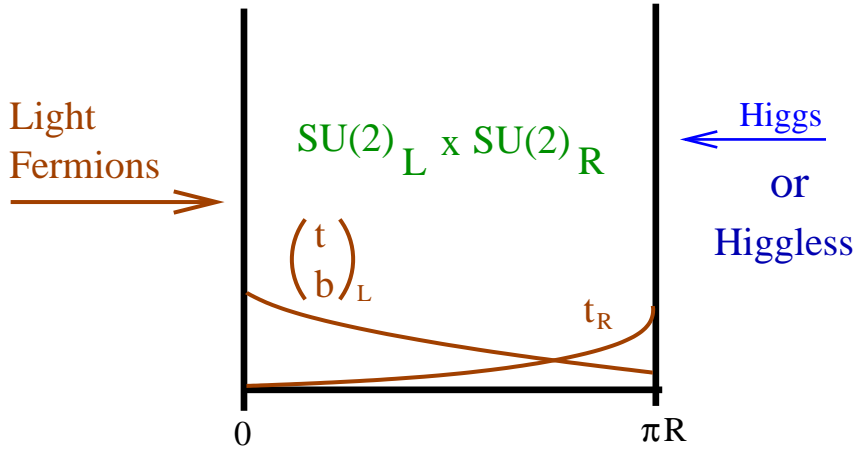

FIG. 10: A Higgless model of EWSB and fermion masses.

allows us to write brane localized vector mass terms such as, for instance

$$
\mathcal{S}_{f}=\int d^{4} x \int d y \delta(y-\pi R) \sqrt{-g}\left\{M_{u} q \psi_{\bar{u}}+M_{d} q \psi_{\bar{d}}+\cdots\right\}
$$

where $M_{u}$ and $M_{d}$ are $\simeq O(1) \mathrm{TeV}$, and $q, \psi_{\bar{u}}$ and $\psi_{\bar{d}}$ a lefthanded, right-handed up and right-handed down quarks respectively. Thus, the fermion mass hierarchy is still generated by $O(1)$ flavor breaking in the bulk fermion mass parameter $c$. The difference now is that the masses are generated by the overlap with a vector mass term as opposed to the Higgs VEV. Thus from Figure 10 we see that the problem of flavor violation coming from the need to have a heavy top quark is still present here [18].

The electroweak constraints on these kinds of theories are quite important. For instance, the $S$ parameter is given by [18, 23]

$$
S \sim 16 \pi \frac{v^{2}}{m_{K K}^{2}}=\frac{N}{\pi},
$$

where in the second equality, $N$ refers to the size of the gauge group in the dual 4D CFT. Thus, for large $N$, which in the $A d S_{5}$ side corresponds to a weakly couple KK sector, the $S$ parameter tends to be larger than experimentally acceptable. Several possibilities have been considered in the literature to deal with this problem. For instance, negative $S$ contributions might be induced by $\mathrm{TeV}$ localized kinetic terms [24], however there is always a constraining combination of $S$ and $T$ [25]. More recently, Ref. [26] advocates peeling light fermions off the Planck brane in order to reduce $S$. This amounts to shift the couplings of light fermions to the gauge bosons, reabsorbing in the process some, or even all, of $S$. Finally, one might take the result in eqn. (19) as an indication that $N$ must be small. This pushes the theory into the realm of a strongly coupled KK sector. This is the scenario entertained in Ref. [18]. The result are theories where the KK sector is not well defined since KK states are not narrow, well spaced resonances. In this case, there is no gap between the TeV scale and the cutoff of the 5D $A d S_{5}$ theory where we could defined individual, weakly coupled states. We would expect one broad resonance encompassing all the KK states. Above the cutoff of a few $\mathrm{TeV}$, stringy dynamics come into play. This scenario is quite reminiscent of a Walking Technicolor theory. This can be seen in the schematic phase diagram of Figure 11, where the 't Hooft coupling $g^{2} N / 16 \pi^{2}$ is plotted against energy. Here, $\Lambda$ is the energy scale where the CFT group exhibits non-trivial IR dynamics. In the large $N$ limit, it is possible to calculate $S$ reliably in the 5D $A d S_{5}$ theory. This is not the case in the Technicolor and Walking Technicolor theories. However, as $N$ is taken to be smaller, reliability may be lost in the 5D theory too. In any case, large or small $N$, the electroweak corrections come mostly from $E \sim \Lambda$, where all theories will give similar results. However, at higher energies the theories may be quite different, with the dual of the $A d S_{5}$, a conformal theory with a 't Hooft coupling above 1 all to way to high energies. The question remains whether or not our knowledge of these differences can be put to use to improve our understanding of strongly coupled theories at the TeV scale. Perhaps, EWSB is a consequence of a $4 \mathrm{D}$ conformal theory and the study of 5D theories could help illuminate some of its technical aspects.

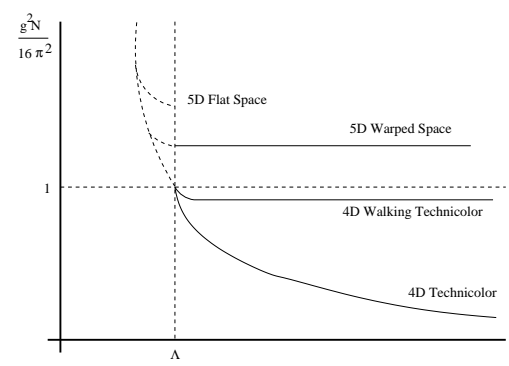

FIG. 11: The 't Hooft coupling vs. energy scale for various theories. From Ref. [18].

In this direction, it is interesting to see how much of QCD at low energies can be obtained by assuming a theory with the IR at the GeV scale, as opposed to TeV. So far the results are remarkably good [27] when it comes to general aspects of the theory, such as the spectrum of vector resonances and their couplings. But we still need to understand why does this work for QCD. As far as we know, QCD is not a conformal or quasi-conformal theory. Why is then well described by RS models in $A d S_{5}$. The aim is to understand how to build strongly coupled theories at the $\mathrm{TeV}$ scale, by making use of weakly coupled theories in the warped background. We may learn something about QCD in the process.

\section{CONCLUSIONS}

The LHC is about to start exploring the energies where we expect the SM to fail. Although in principle is possible to have a weak scale only completed with the SM light Higgs, this is very unnatural. More natural completions of the electroweak theory at the $\mathrm{TeV}$ scale require the cancellation of the quadratic divergences in the Higgs mass.

Theories with extra spatial dimensions provide alternatives to supersymmetry and strong dynamics at the $\mathrm{TeV}$ scale. We 
reviewed the main three ways in which these can solve the gauge hierarchy problem. In the case of Large Extra Dimensions, the true fundamental scale of gravity is the $\mathrm{TeV}$ scale, whereas the Planck scale is derived from a volume suppression. Experimental signatures at colliders are mainly the observation of various missing $E_{T}$ channels corresponding to the collective graviton states.

Theories with Universal Extra Dimensions can have compactification radii still somewhat smaller than the TeV. In them the fundamental scale of quantum gravity might reside in the tens of TeV. They present a very rich, albeit model dependent, phenomenology.

Finally, the Randall-Sundrum scenario, where the extra dimension has a curved geometry corresponding to $A d S_{5}$, may also give rise to a theory of flavor if matter and gauge fields are allowed to propagate in the bulk. Various signals, both for its KK spectrum, as well as for flavor violating interactions related to the origin of the flavor hierarchy, would be observed at the LHC. These kinds of extra dimensional theories could be viewed as a way to build strongly coupled 4D theories of the $\mathrm{TeV}$. Understanding the relation between a weakly coupled theory in $A d S_{5}$ and a strongly coupled theory in 4D should be an important step towards these models. Perhaps, modeling QCD at low energies in the $A d S_{5}$ framework will provide us with some clues on how to go about studying a strongly coupled spectrum at the $\mathrm{TeV}$, in the event this is what is observed at the LHC.
[1] S. P. Martin, "A Supersymmetry Primer", [arXiv:hepph/9709356], in Kane, G.L. (ed.): Perspectives on supersymmetry 1-98.

[2] C. T. Hill and E. H. Simmons, Phys. Rept. 381, 235 (2003) [Erratum-ibid. 390, 553 (2004)] [arXiv:hep-ph/0203079].

[3] N. Arkani-Hamed, S. Dimopoulos, and G. R. Dvali, Phys. Lett. B 429, 263 (1998) [arXiv:hep-ph/9803315];N. Arkani-Hamed, S. Dimopoulos, and G. R. Dvali, Phys. Rev. D 59, 086004 (1999) [arXiv:hep-ph/9807344]

[4] T. Appelquist, H. C. Cheng, and B. A. Dobrescu, Phys. Rev. D 64, 035002 (2001) [arXiv:hep-ph/0012100].

[5] L. Randall and R. Sundrum, Phys. Rev. Lett. 83, 3370 (1999) [arXiv:hep-ph/9905221]

[6] M. Karagoz, these proceedings

[7] D. M. Eardley and S. B. Giddings, Phys. Rev. D 66, 044011 (2002) [arXiv:gr-qc/0201034].

[8] L. A. Anchordoqui, J. L. Feng, H. Goldberg, and A. D. Shapere, Phys. Rev. D 65, 124027 (2002) [arXiv:hep-ph/0112247].

[9] H. C. Cheng, K. T. Matchev, and M. Schmaltz, Phys. Rev. D 66 (2002) 036005 [arXiv:hep-ph/0204342].

[10] H. C. Cheng, K. T. Matchev, and M. Schmaltz, Phys. Rev. D 66 (2002) 056006 [arXiv:hep-ph/0205314].

[11] T. Appelquist, B. A. Dobrescu, E. Ponton, and H. U. Yee, Phys. Rev. Lett. 87, 181802 (2001) [arXiv:hep-ph/0107056].

[12] B. A. Dobrescu and E. Ponton, JHEP 0403, 071 (2004) [arXiv:hep-th/0401032].

[13] G. Burdman, B. A. Dobrescu, and E. Ponton, JHEP 0602, 033 (2006) [arXiv:hep-ph/0506334].

[14] G. Burdman, B. A. Dobrescu, and E. Ponton, arXiv:hep$\mathrm{ph} / 0601186$.
[15] H. Davoudiasl, J. L. Hewett, and T. G. Rizzo, Phys. Rev. D 63, 075004 (2001) [arXiv:hep-ph/0006041].

[16] K. Agashe, A. Delgado, M. J. May, and R. Sundrum, JHEP 0308, 050 (2003) [arXiv:hep-ph/0308036].

[17] T. Gherghetta and A. Pomarol, Nucl. Phys. B 586, 141 (2000) [arXiv:hep-ph/0003129].

[18] G. Burdman and Y. Nomura, Phys. Rev. D 69, 115013 (2004) [arXiv:hep-ph/0312247].

[19] G. Burdman, Phys. Lett. B 590, 86 (2004) [arXiv:hep$\mathrm{ph} / 0310144]$.

[20] C. Csaki, C. Grojean, H. Murayama, L. Pilo, and J. Terning, Phys. Rev. D 69, 055006 (2004) [arXiv:hep-ph/0305237].

[21] R. Sekhar Chivukula, D. A. Dicus, and H. J. He, Phys. Lett. B 525, 175 (2002) [arXiv:hep-ph/0111016].

[22] C. Csaki, C. Grojean, L. Pilo, and J. Terning, Phys. Rev. Lett. 92, 101802 (2004) [arXiv:hep-ph/0308038].

[23] R. Barbieri, A. Pomarol, and R. Rattazzi, Phys. Lett. B 591, 141 (2004) [arXiv:hep-ph/0310285].

[24] G. Cacciapaglia, C. Csaki, C. Grojean, and J. Terning, arXiv:hep-ph/0401160.

[25] R. S. Chivukula, E. H. Simmons, H. J. He, M. Kurachi, and M. Tanabashi, arXiv:hep-ph/0406077.

[26] G. Cacciapaglia, C. Csaki, C. Grojean, and J. Terning, arXiv:hep-ph/0409126.

[27] J. Erlich, E. Katz, D. T. Son, and M. A. Stephanov, Phys. Rev. Lett. 95, 261602 (2005) [arXiv:hep-ph/0501128];

L. Da Rold and A. Pomarol, Nucl. Phys. B 721, 79 (2005) [arXiv:hep-ph/0501218]. 